\title{
含三元杂环席夫碱类衍生物的合成及生物活性研究
}

熊 壮 李海畅梁娜 尹 娟卢平薛伟*

(贵州大学精细化工研究开发中心 教育部绿色农药与农业生物工程重点实验室 贵阳 550025)

\begin{abstract}
摘要 设计合成了 10 个新型的含三元杂环席夫碱类衍生物, 所有化合物结构通过 ${ }^{1} \mathrm{H} \mathrm{NMR},{ }^{13} \mathrm{C} N \mathrm{NR}, \mathrm{IR}$ 和元素分析确 证, 生物活性初步研究结果表明, 在 $50 \mathrm{mg} / \mathrm{L}$ 浓度下, 目标化合物对小麦赤霉菌 $(G$. zeae)、苹果腐烂病菌 $(C$. mandshurica) 和辣椒枯萎病菌(F. oxysporum) 具有一定的抑制活性.
\end{abstract}

关键词 三唑; 哒嗪酮; 生物活性; 合成

\section{Synthesis and Bioactivity of Three-Ring Heterocyclic Schiff Base Derivatives}

\author{
Xiong, Zhuang Li, Haichang $\quad$ Liang, $\mathrm{Na} \quad$ Yin, Juan $\quad$ Lu, Ping $\quad$ Xue, Wei* \\ (Key Laboratory of Green Pesticide and Bioengineering, Ministry of Education, Research and Development Center for \\ Fine Chemicals, Guizhou University, Guiyang 550025)
}

\begin{abstract}
Ten novel three-ring heterocyclic Schiff-base derivatives were designed and synthesized. Their structures of all these compounds were confirmed by ${ }^{1} \mathrm{H} N \mathrm{NM},{ }^{13} \mathrm{C} \mathrm{NMR}$, IR spectra and elemental analysis. The preliminary result of bioassay showed that the title compounds exhibited some degree of antifungal activities on G. zeae, C. mandshurica and F. oxysporum under $50 \mathrm{mg} / \mathrm{L}$ concentration.
\end{abstract}

Keywords triazole; pyridazinone; bioactivity; synthesis

哒嗪类化合物是一类具有广泛生物活性的杂环化 合物, 如高效除草、杀虫、杀螨、植物生长调节等活性, 并且具有低毒、低残留的特点 ${ }^{[1,2]}$. 目前, 已有许多商品 化的哒嗪类农药, 相继开发出了哒溙硫磷杀虫剂、哒溙 酮杀螨剂、哒菌清杀菌剂 ${ }^{[3]}$, 在病虫害的综合防治和降 低农药对环境污染方面发挥着重要作用.

1,2,4-三唑类化合物也是一类具有良好生物活性的 杂环化合物 ${ }^{[4,5]}$, 在医药和农药中有着广泛的应用, 在医 药上具有抗菌、抗痉挛、消炎、抗血小板凝聚等功能 ${ }^{[6,7]}$; 在农药上具有杀虫、杀菌、除草和植物生长调节等活 性 $^{[8 \sim 10]}$, 商品化的杀菌剂中有很多含有三唑结构, 如三 唑酮、烯唑醇和腈菌唑等.

吡啶环在农药合成中也是一个十分重要的含氮杂 环, 吡啶类衍生物中不仅有杀虫剂、除草剂, 而且有高 效的杀菌剂, 如啶酰菌胺和啶氧菌酯 ${ }^{[1]}$. 此外, 席夫碱
类化合物具有多种生物活性, 如抑菌、杀菌、抗病毒、 抗肿瘤等生物活性 ${ }^{[12]}$. 为了篮选新的杀菌剂, 本课题组 根据活性拼接原理，以 2,3-二氯吡啶和糠氯酸为原料合 成了含吡啶环的 3(2H)-哒嗪酮; 以没食子酸为起始原料 经过酯化、醚化、肼解和合环等步骤合成了 4-氨基5-(3,4,5-三甲氧基苯基)-1,2,4-三唑-3-硫酮，进而与醛缩 合生成含席夫碱结构的三唑类化合物，最后以乙腈为溶 剂，在碳酸钾的作用，将两者以硫醚的形式拼接在一起， 设计合成了 10 个含吡啶环、哒嗪环和三唑环的三元杂 环化合物, 化合物的结构经过 ${ }^{1} \mathrm{H} N \mathrm{NMR},{ }^{13} \mathrm{C}$ NMR, IR 和 元素分析的确证，并以小麦赤霉菌、苹果腐烂菌和辣椒 枯萎菌为供试菌种对其进行了抑菌活性测试，以期发现 具有潜在杀菌活性的先导化合物, 为开发高效、低毒和 环境友好的杀菌剂提供可能. 合成路线见 Scheme 1.

\footnotetext{
*E-mail: wxue@gzu.edu.cn

Received December 24, 2011; revised February 26, 2012; published online April 11, 2012.

Project supported by the National Natural Science Foundation of China (No. 21062005), and the Innovation Foundation for Postgraduate Students in Guizhou University (No. 2011033).

国家自然科学基金(No. 21062005)和贵州大学研究生创新基金(No. 2011033)资助项目.
} 

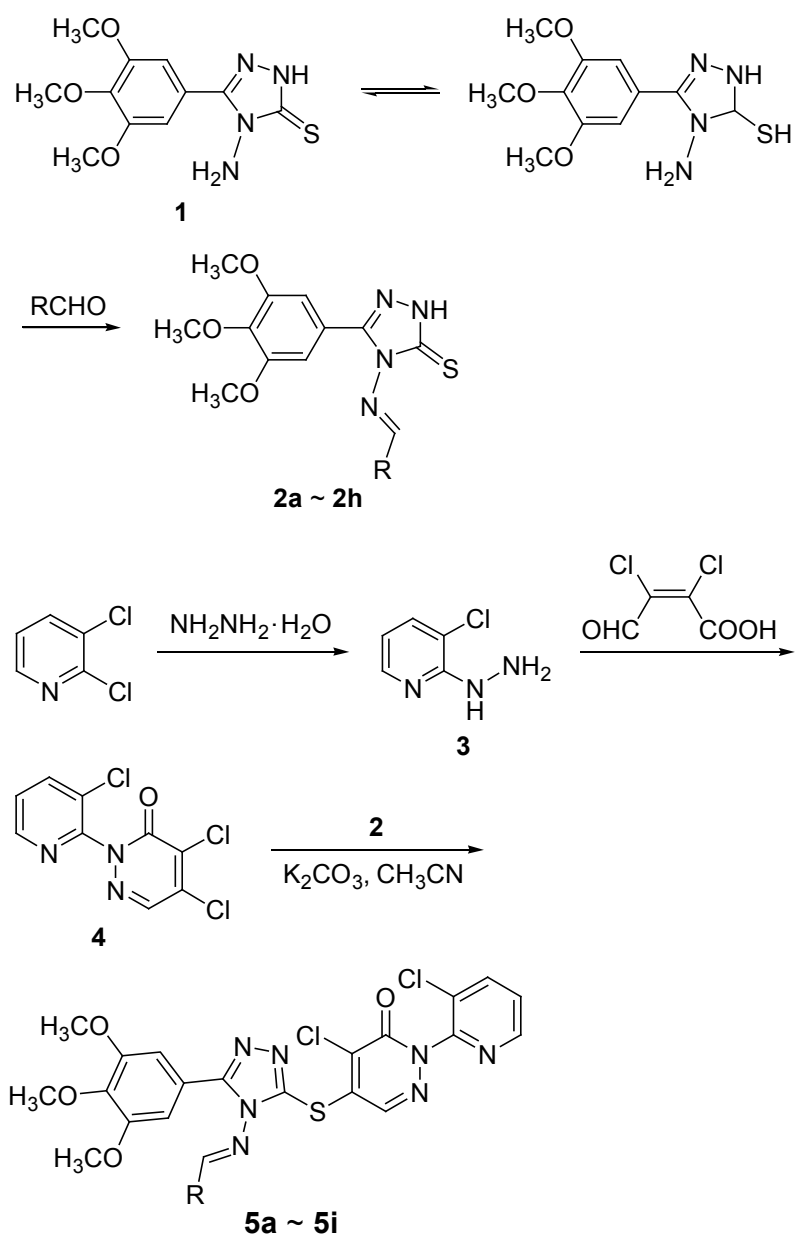

R: 4- $\mathrm{FC}_{6} \mathrm{H}_{4}(\mathbf{5 a}), 4-\mathrm{ClC}_{6} \mathrm{H}_{4}(\mathbf{5 b}), \mathrm{C}_{6} \mathrm{H}_{5}(\mathbf{5 c}), 4-\mathrm{CH}_{3} \mathrm{OC}_{6} \mathrm{H}_{4}(\mathbf{5 d})$,

2,3- $\left(\mathrm{CH}_{3} \mathrm{O}\right)_{2} \mathrm{C}_{6} \mathrm{H}_{3}(\mathbf{5 e}), 2-\mathrm{FC}_{6} \mathrm{H}_{4}(\mathbf{5 f}), 3-\mathrm{FC}_{6} \mathrm{H}_{4}(\mathbf{5 g})$,

2,5- $\left(\mathrm{CH}_{3} \mathrm{O}\right)_{2} \mathrm{C}_{6} \mathrm{H}_{3}(5 \mathbf{h}), \mathrm{C}_{6} \mathrm{H}_{4} \mathrm{CH}_{2}=\mathrm{CH}_{2}(\mathbf{5 i}), 2-\mathrm{ClC}_{6} \mathrm{H}_{4}(\mathbf{5 j})$

\section{Scheme 1}

\section{1 实验部分}

\section{1 仪器与试剂}

JEOL-ECX 500 NMR 核磁共振仪(日本电子), TMS 作内标, DMSO- $d_{6}$ 为溶剂; Elementar Vario-III 型元素分 析仪(德国); IR Prestige-21 型红外光谱仪(日本岛津公 司), $\mathrm{KBr}$ 压片; X-5 型熔点测定仪(温度计未校正). 所用 试剂均为分析纯；4-氨基-5-(3,4,5-三甲氧基苯基)-1,2,4三唑-3-硫酮(1)参照文献[13，14]制备; 4-取代苯次甲亚 胺-5-(3,4,5-三甲氧基苯基)-4H-1,2,4-三唑-3-硫酮(2)参照 文献[9]制备.

\section{2 中间体 2-(3-氯-吡啶-2-基)-4,5-二氯-3(2H)-哒嗪酮} (4)的合成

将 $0.1 \mathrm{~mol} 2,3$-二氯吡啶加入至 $250 \mathrm{~mL}$ 的三口瓶中, 然后加入 $100 \mathrm{~mL}$ 的 $80 \%$ 水合肼溶液, 加热回流 $5 \mathrm{~h}$, TLC 跟踪至反应完全, 冷却至室温, 析出大量白色针状 晶体，抽滤得产物 3, 收率 $95 \%$. m.p. 169 171 ${ }^{\circ} \mathrm{C}$ (文献
值 $\left.{ }^{[15]} 168{ }^{\circ} \mathrm{C}\right)$.

将 $10 \mathrm{mmol}$ 糠氯酸加入至 $250 \mathrm{~mL}$ 的三口瓶中, 加 入 $100 \mathrm{~mL}$ 的甲醇, 摚拌至溶液呈澄清, 然后加入 10 $\mathrm{mmol}$ 的化合物 $\mathbf{3}$, 加入 $8 \mathrm{~mL}$ 的 $5 \%$ 稀盐酸溶液, 加热回 流 $3 \mathrm{~h}$, TLC 跟踪至反应完全, 减压蒸去溶剂, 得大量黄 色固体, 用无水乙醇重结晶, 得产物 4 . 白色固体粉末, 收率 80.8\%. m.p. 201 202 ${ }^{\circ} \mathrm{C} ;{ }^{1} \mathrm{H}$ NMR (DMSO- $d_{6}, 500$ MHz) $\delta: 8.59$ (t, $\left.J=1.70 \mathrm{~Hz}, 1 \mathrm{H}, \mathrm{C}_{6} \mathrm{H}_{3} \mathrm{NH}\right), 8.41$ (d, $J=$ $\left.2.30 \mathrm{~Hz}, 1 \mathrm{H}, \mathrm{C}_{6} \mathrm{H}_{3} \mathrm{NH}\right), 8.27(\mathrm{~d}, J=8.05 \mathrm{~Hz}, 1 \mathrm{H}$, $\left.\mathrm{C}_{6} \mathrm{H}_{3} \mathrm{NH}\right), 7.66 \sim 7.68(\mathrm{~m}, 1 \mathrm{H}, \mathrm{CH}=\mathrm{NN}) ;{ }^{13} \mathrm{C} \mathrm{NMR}$ (DMSO- $\left.d_{6}, 125 \mathrm{MHz}\right) \delta: 155.5,149.4,148.6,140.5,138.1$, 137.1, 134.4, 128.5, 127.9; IR (KBr) v: 3081, 1664, 1646, $1592,1461 \mathrm{~cm}^{-1}$.

\section{3 目标化合物 5 的合成}

将 $1.5 \mathrm{mmol}$ 化合物 $2 、 1.5 \mathrm{mmol}$ 的碳酸钾和 $30 \mathrm{~mL}$ 乙腈置于 $100 \mathrm{~mL}$ 反应瓶中, 室温搅拌 $15 \mathrm{~min}$ 后, 加入 $1.5 \mathrm{mmol}$ 的 4 在 $45{ }^{\circ} \mathrm{C}$ 下反应 $2 \mathrm{~h}, \mathrm{TLC}$ 跟踪至反应完全, 过滤, 反应液倒入冷水中, 析出固体, 抽滤, 烘干, 固体 经硅胶柱层析 $[V$ (石油醚 $): V($ 乙酸乙酯 $)=1: 1]$ 分离纯 化, 得产物 $\mathbf{5 a} \sim \mathbf{5}$ j.

(E)-4-氯-2-(3-氯吡啶-2-基)-5-((4-((4-氟苯基次甲基) 氨基)-5-(3,4,5-三甲氧基苯基)-4H-1,2,4-三唑-3-基)硫 基)-3(2H)-哒嗪酩(5a)：淡黄色粉末，产率 41.5\%. m.p. $123 \sim 125{ }^{\circ} \mathrm{C} ;{ }^{1} \mathrm{H}$ NMR (DMSO- $\left.d_{6}, 500 \mathrm{MHz}\right) \delta$ : 9.03 (s, $1 \mathrm{H}, \mathrm{CH}=\mathrm{N}), 8.56 \sim 8.58\left(\mathrm{~m}, 1 \mathrm{H}, \mathrm{C}_{6} \mathrm{H}_{3} \mathrm{NH}\right), 8.23 \sim 8.25$ $\left(\mathrm{m}, 1 \mathrm{H}, \mathrm{C}_{6} \mathrm{H}_{3} \mathrm{NH}\right), 7.96 \sim 7.99\left(\mathrm{~m}, 2 \mathrm{H}, 4-\mathrm{FC}_{6} \mathrm{H}_{4}\right), 7.72(\mathrm{~s}$, $1 \mathrm{H}, \mathrm{CH}=\mathrm{NN}), 7.64 \sim 7.67\left(\mathrm{~m}, 1 \mathrm{H}, \mathrm{C}_{6} \mathrm{H}_{3} \mathrm{NH}\right), 7.39 \sim 7.42$ $\left(\mathrm{m}, 2 \mathrm{H}, 4-\mathrm{FC}_{6} \mathrm{H}_{4}\right), 7.21\left[\mathrm{~s}, 2 \mathrm{H},\left(\mathrm{CH}_{3} \mathrm{O}\right)_{3} \mathrm{C}_{6} \mathrm{H}_{2}\right], 3.71[\mathrm{~s}, 6 \mathrm{H}$, $\left.\left(\mathrm{OCH}_{3}\right)_{2}\right], 3.68\left(\mathrm{~s}, 3 \mathrm{H}, \mathrm{OCH}_{3}\right) ;{ }^{13} \mathrm{C}$ NMR (DMSO- $d_{6}, 125$ MHz) $\delta: 170.6,165.0,154.3,153.4,152.4,149.4,148.6$, $140.4,139.9,139.7,135.2,132.4,131.2,128.5,128.3$, $127.8,121.5,117.4,117.2,106.1,60.5,55.9$; IR $(\mathrm{KBr}) v$ : 3018, 2933, 2835, 1732, 1693, 1672, 1600, 1510, 1477, $1429,1283,734 \mathrm{~cm}^{-1}$. Anal. calcd for $\mathrm{C}_{27} \mathrm{H}_{20} \mathrm{Cl}_{2} \mathrm{FN}_{7} \mathrm{O}_{4} \mathrm{~S}$ : C 51.60, H 3.21, N 15.60; found C 51.80, H 3.33, N 15.86.

(E)-4-氯-2-(3-氯吡啶-2-基)-5-((4-((4-氯苯基次甲基) 氨基)-5-(3,4,5-三甲氧基苯基)-4H-1,2,4-三唑-3-基)硫 基)-3(2H)-哒嗪酮 (5b): 白色粉末，产率 39.2\%. m.p. $149 \sim 151{ }^{\circ} \mathrm{C} ;{ }^{1} \mathrm{H}$ NMR (DMSO- $\left.d_{6}, 500 \mathrm{MHz}\right) \delta: 9.07$ (s, $1 \mathrm{H}, \mathrm{CH}=\mathrm{N}), 8.61\left(\mathrm{~d}, J=5.15 \mathrm{~Hz}, 1 \mathrm{H}, \mathrm{C}_{6} \mathrm{H}_{3} \mathrm{NH}\right), 8.28(\mathrm{~d}$, $\left.J=6.30 \mathrm{~Hz}, 1 \mathrm{H}, \mathrm{C}_{6} \mathrm{H}_{3} \mathrm{NH}\right), 7.95(\mathrm{~d}, J=8.55 \mathrm{~Hz}, 2 \mathrm{H}$, 4- $\left.\mathrm{ClC}_{6} \mathrm{H}_{4}\right), 7.67\left(\mathrm{~d}, J=8.55 \mathrm{~Hz}, 2 \mathrm{H}, 4-\mathrm{ClC}_{6} \mathrm{H}_{4}\right), 7.76(\mathrm{~s}$, $1 \mathrm{H}, \mathrm{CH}=\mathrm{NN}), 7.70\left(\mathrm{t}, J=4.55 \mathrm{~Hz}, 1 \mathrm{H}, \mathrm{C}_{6} \mathrm{H}_{3} \mathrm{NH}\right), 7.67(\mathrm{~d}$, $\left.J=8.55 \mathrm{~Hz}, 2 \mathrm{H}, 4-\mathrm{ClC}_{6} \mathrm{H}_{4}\right), 7.24\left[\mathrm{~s}, 2 \mathrm{H},\left(\mathrm{CH}_{3} \mathrm{O}\right)_{3} \mathrm{C}_{6} \mathrm{H}_{2}\right]$, $3.75\left[\mathrm{~s}, 6 \mathrm{H},\left(\mathrm{OCH}_{3}\right)_{2}\right], 3.72\left(\mathrm{~s}, 3 \mathrm{H}, \mathrm{OCH}_{3}\right) ;{ }^{13} \mathrm{C} \mathrm{NMR}$ 
(DMSO- $\left.d_{6}, 125 \mathrm{MHz}\right) \delta: 170.4,154.3,153.5,152.4,149.5$, $148.6,140.5,139.9,139.8,139.7,139.2,135.3,131.4$, $131.3,130.5,130.2,128.5,127.9,121.5,106.3,60.7,56.4$; IR (KBr) v: 3075, 2931, 2831, 1714, 1680, 1674, 1633, 1589, 1475, 1429, 1242, $735 \mathrm{~cm}^{-1}$. Anal. calcd for $\mathrm{C}_{27} \mathrm{H}_{20} \mathrm{Cl}_{3} \mathrm{~N}_{7} \mathrm{O}_{4} \mathrm{~S}$ : C 50.28, H 3.13, N 15.20; found C 50.53, $\mathrm{H} 3.43, \mathrm{~N} 15.53$.

(E)-4-氯-2-(3-氯吡啶-2-基)-5-((4-((苯基次甲基)氨 基 )-5-(3,4,5-三甲氧基苯基)-4H-1,2,4-三唑-3-基)硫 基)-3(2H)-哒嗪酮(5c): 淡黄色粉末, 产率 41.5\%. m.p. $199 \sim 201{ }^{\circ} \mathrm{C} ;{ }^{1} \mathrm{H}$ NMR (DMSO- $\left.d_{6}, 500 \mathrm{MHz}\right) \delta: 9.03$ (s, $1 \mathrm{H}, \mathrm{CH}=\mathrm{N}), 8.56\left(\mathrm{~d}, J=5.15 \mathrm{~Hz}, 1 \mathrm{H}, \mathrm{C}_{6} \mathrm{H}_{3} \mathrm{NH}\right), 8.23$ (d, $\left.J=6.90 \mathrm{~Hz}, 1 \mathrm{H}, \mathrm{C}_{6} \mathrm{H}_{3} \mathrm{NH}\right), 7.88(\mathrm{~d}, J=7.40 \mathrm{~Hz}, 2 \mathrm{H}$, $\left.\mathrm{C}_{6} \mathrm{H}_{5} \mathrm{H}\right), 7.73(\mathrm{~s}, 1 \mathrm{H}, \mathrm{CH}=\mathrm{NN}), 7.64 \sim 7.67(\mathrm{~m}, 2 \mathrm{H}$, $\left.\mathrm{C}_{6} \mathrm{H}_{3} \mathrm{NH}+\mathrm{C}_{6} \mathrm{H}_{5} \mathrm{H}\right), 7.55\left(\mathrm{t}, J=7.45 \mathrm{~Hz}, 2 \mathrm{H}, \mathrm{C}_{6} \mathrm{H}_{5} \mathrm{H}\right), 7.22$ [s, 2H, $\left(\mathrm{CH}_{3} \mathrm{O}\right)_{3} \mathrm{C}_{6} \mathrm{H}_{2}$ ], 3.71 [s, 6H, $\left.\left(\mathrm{OCH}_{3}\right)_{2}\right], 3.67(\mathrm{~s}, 3 \mathrm{H}$, $\left.\mathrm{OCH}_{3}\right) ;{ }^{13} \mathrm{C}$ NMR (DMSO- $\left.d_{6}, 125 \mathrm{MHz}\right) \delta: 171.9,154.2$, $153.5,152.4,149.5,148.6,140.4,139.9,139.7,139.6$, $135.2,134.5,131.6,131.1,130.0,129.6,128.5,127.8$, 121.5, 106.1, 60.6, 56.4; IR (KBr) v: 3061, 2929, 2835, $1730,1687,1674,1611,1583,1479,1423,1236,731$ $\mathrm{cm}^{-1}$. Anal. calcd for $\mathrm{C}_{27} \mathrm{H}_{21} \mathrm{Cl}_{2} \mathrm{~N}_{7} \mathrm{O}_{4} \mathrm{~S}$ : C 53.12, H 3.47, N 16.06; found $\mathrm{C} 53.43, \mathrm{H} 3.62, \mathrm{~N} 16.34$.

(E)-4-氯-2-(3-氯吡啶-2-基)-5-((4-((4-甲氧基苯基次 甲基)氨基)-5-(3,4,5-三甲氧基苯基)- $4 H-1,2,4$-三唑-3-基) 硫基)-3(2H)-哒嗪酮(5d): 淡黄色粉末, 产率 43.3\%. m.p. $163 \sim 165{ }^{\circ} \mathrm{C} ;{ }^{1} \mathrm{H}$ NMR (DMSO- $\left.d_{6}, 500 \mathrm{MHz}\right) \delta: 8.91$ (s, $1 \mathrm{H}, \mathrm{CH}=\mathrm{N}), 8.57\left(\mathrm{~d}, J=4.55 \mathrm{~Hz}, 1 \mathrm{H}, \mathrm{C}_{6} \mathrm{H}_{3} \mathrm{NH}\right), 8.24$ (d, $\left.J=8.05 \mathrm{~Hz}, 1 \mathrm{H}, \mathrm{C}_{6} \mathrm{H}_{3} \mathrm{NH}\right), 7.84(\mathrm{~d}, J=8.60 \mathrm{~Hz}, 2 \mathrm{H}$, $\left.4-\mathrm{CH}_{3} \mathrm{OC}_{6} \mathrm{H}_{4}\right), 7.71(\mathrm{~s}, 1 \mathrm{H}, \mathrm{CH}=\mathrm{NN}), 7.66(\mathrm{t}, J=6.55 \mathrm{~Hz}$, $\left.1 \mathrm{H}, \mathrm{C}_{6} \mathrm{H}_{3} \mathrm{NH}\right), 7.23$ [s, 2H, $\left.\left(\mathrm{CH}_{3} \mathrm{O}\right)_{3} \mathrm{C}_{6} \mathrm{H}_{2}\right], 7.09(\mathrm{~d}, J=8.60$ $\left.\mathrm{Hz}, 2 \mathrm{H}, 4-\mathrm{CH}_{3} \mathrm{OC}_{6} \mathrm{H}_{4}\right), 3.82\left(\mathrm{~s}, 3 \mathrm{H}, \mathrm{OCH}_{3}\right), 3.70[\mathrm{~s}, 6 \mathrm{H}$, $\left.\left(\mathrm{OCH}_{3}\right)_{2}\right], 3.67\left(\mathrm{~s}, 3 \mathrm{H}, \mathrm{OCH}_{3}\right) ;{ }^{13} \mathrm{C} \mathrm{NMR}$ (DMSO- $d_{6}, 125$ MHz) $\delta: 171.23,154.6,153.2,152.4,149.6,148.8,140.5$, $139.9,139.7,135.3,131.9,130.9,128.7,127.9,123.9$, 121.6, 115.5, 106.0, 60.7, 60.2, 56.3; IR (KBr) v: 3021, 2931, 2833, 1735, 1693, 1666, 1598, 1556, 1514, 1427, $1238,744 \mathrm{~cm}^{-1}$. Anal. calcd for $\mathrm{C}_{28} \mathrm{H}_{23} \mathrm{Cl}_{2} \mathrm{~N}_{7} \mathrm{O}_{5} \mathrm{~S}$ : C 52.51, H 3.62, N 15.31; found C 52.60, H 3.91, N 15.64.

(E)-4-氯-2-(3-氯吡啶-2-基)-5-((4-((2,3-甲氧基苯基 次甲基)氨基)-5-(3,4,5-三甲氧基苯基)-4H-1,2,4-三唑-3基)硫基)-3(2H)-哒嗪酮(5e)：淡黄色粉末，产率 44.5\%. m.p. $203 \sim 205{ }^{\circ} \mathrm{C}$; ${ }^{1} \mathrm{H}$ NMR (DMSO- $\left.d_{6}, 500 \mathrm{MHz}\right) \delta$ : $8.98(\mathrm{~s}, 1 \mathrm{H}, \mathrm{CH}=\mathrm{N}), 8.55\left(\mathrm{~d}, J=4.60 \mathrm{~Hz}, 1 \mathrm{H}, \mathrm{C}_{6} \mathrm{H}_{3} \mathrm{NH}\right)$, $8.22\left(\mathrm{~d}, J=8.00 \mathrm{~Hz}, 1 \mathrm{H}, \mathrm{C}_{6} \mathrm{H}_{3} \mathrm{NH}\right), 7.78(\mathrm{~s}, 1 \mathrm{H}, \mathrm{CH}=$
$\mathrm{NN}), 7.61 \sim 7.69\left[\mathrm{~m}, 2 \mathrm{H}, \mathrm{C}_{6} \mathrm{H}_{3} \mathrm{NH}+\left(\mathrm{CH}_{3} \mathrm{O}\right)_{2} \mathrm{C}_{6} \mathrm{H}_{3}\right], 7.37$ $\left[\mathrm{d}, J=3.45 \mathrm{~Hz}, 1 \mathrm{H},\left(\mathrm{CH}_{3} \mathrm{O}\right)_{2} \mathrm{C}_{6} \mathrm{H}_{3}\right], 7.21\left[\mathrm{~s}, 2 \mathrm{H},\left(\mathrm{CH}_{3} \mathrm{O}\right)_{3^{-}}\right.$ $\mathrm{C}_{6} \mathrm{H}_{2}$ ], $7.11\left[\mathrm{~d}, J=9.15 \mathrm{~Hz}, 1 \mathrm{H},\left(\mathrm{CH}_{3} \mathrm{O}\right)_{2} \mathrm{C}_{6} \mathrm{H}_{3}\right], 3.73[\mathrm{~s}$, $\left.6 \mathrm{H},\left(\mathrm{OCH}_{3}\right)_{2}\right], 3.70\left(\mathrm{~s}, 3 \mathrm{H}, \mathrm{OCH}_{3}\right), 3.69$ [s, 6H, $\left.\left(\mathrm{OCH}_{3}\right)_{2}\right]$; ${ }^{13} \mathrm{C}$ NMR (DMSO- $\left.d_{6}, 125 \mathrm{MHz}\right) \delta: 165.3,154.9,154.3$, $153.7,153.5,152.6,149.5,148.6,140.4,139.8,139.2$, $135.1,132.2,130.8,129.2,128.5,127.9,123.1,121.8$, $114.8,109.9,106.4,65.58,57.00,56.4,56.1$; IR (KBr) $v$ : 3091, 2935, 2835, 1721, 1689, 1674, 1587, 1571, 1481, $1425,1128,732 \mathrm{~cm}^{-1}$. Anal. calcd for $\mathrm{C}_{29} \mathrm{H}_{25} \mathrm{Cl}_{2} \mathrm{~N}_{7} \mathrm{O}_{6} \mathrm{~S}: \mathrm{C}$ 51.95, H 3.76, N 14.62; found C 51.98, H 3.83, N 14.83.

(E)-4-氯-2-(3-氯吡啶-2-基)-5-((4-((2-氟苯基次甲基) 氨基)-5-(3,4,5-三甲氧基苯基)-4H-1,2,4-三唑-3-基)硫 基)-3(2H)-哒嗪酮(5f): 淡黄色粉末，产率 46.6\%. m.p. $222 \sim 224{ }^{\circ} \mathrm{C} ;{ }^{1} \mathrm{H}$ NMR (DMSO- $\left.d_{6}, 500 \mathrm{MHz}\right) \delta$ : 9.19 (s, $1 \mathrm{H}, \mathrm{CH}=\mathrm{N}), 8.60\left(\mathrm{~d}, J=4.55 \mathrm{~Hz}, 1 \mathrm{H}, \mathrm{C}_{6} \mathrm{H}_{3} \mathrm{NH}\right), 8.27(\mathrm{~d}$, $\left.J=8.00 \mathrm{~Hz}, 1 \mathrm{H}, \mathrm{C}_{6} \mathrm{H}_{3} \mathrm{NH}\right), 8.05$ (t, $J=7.45 \mathrm{~Hz}, 1 \mathrm{H}$, $\left.\mathrm{C}_{6} \mathrm{H}_{3} \mathrm{NH}\right), 7.69(\mathrm{~s}, 1 \mathrm{H}, \mathrm{CH}=\mathrm{NN}), 7.68 \sim 7.69(\mathrm{~m}, 2 \mathrm{H}$, $\left.2-\mathrm{FC}_{6} \mathrm{H}_{4}\right), 7.40 \sim 7.47\left(\mathrm{~m}, 2 \mathrm{H}, 2-\mathrm{FC}_{6} \mathrm{H}_{4}\right), 7.26[\mathrm{~s}, 2 \mathrm{H}$, $\left(\mathrm{CH}_{3} \mathrm{O}\right)_{3} \mathrm{C}_{6} \mathrm{H}_{2}$ ], 3.77 [s, 6H, $\left.\left(\mathrm{OCH}_{3}\right)_{2}\right], 3.73$ (s, 3H, $\left.\mathrm{OCH}_{3}\right)$; ${ }^{13} \mathrm{C}$ NMR (DMSO- $\left.d_{6}, 125 \mathrm{MHz}\right) \delta$ : 164.9, 163.5, 161.4, $154.2,153.4,152.5,149.5,148.6,140.4,139.9,139.8$, $139.6,136.8,135.1,131.1,128.6,127.9,126.1,121.6$, 119.4, 117.2, 106.2, 60.8, 56.3; IR (KBr) v: 3013, 2933, 2829, 1730, 1693, 1676, 1614, 1585, 1465, 1423, 1238, $775 \mathrm{~cm}^{-1}$. Anal. calcd for $\mathrm{C}_{27} \mathrm{H}_{20} \mathrm{Cl}_{2} \mathrm{FN}_{7} \mathrm{O}_{4} \mathrm{~S}$ : C $51.60, \mathrm{H}$ 3.21, N 15.60; found C 51.91, H 3.53, N 15.82.

(E)-4-氯-2-(3-氯吡啶-2-基)-5-((4-((3-氟苯基次甲基) 氨基)-5-(3,4,5-三甲氧基苯基)-4H-1,2,4-三唑-3-基)硫 基)-3(2H)-哒嗪酮(5g): 淡黄色粉末，产率 46.1\%. m.p. 213 $215{ }^{\circ} \mathrm{C} ;{ }^{1} \mathrm{H}$ NMR (DMSO- $\left.d_{6}, 500 \mathrm{MHz}\right) \delta$ : 9.04 (s, $1 \mathrm{H}, \mathrm{CH}=\mathrm{N}), 8.57\left(\mathrm{~d}, J=4.55 \mathrm{~Hz}, 1 \mathrm{H}, \mathrm{C}_{6} \mathrm{H}_{3} \mathrm{NH}\right), 8.24(\mathrm{~d}$, $\left.J=8.00 \mathrm{~Hz}, 1 \mathrm{H}, \mathrm{C}_{6} \mathrm{H}_{3} \mathrm{NH}\right), 7.50 \sim 7.75\left[\mathrm{~m}, 6 \mathrm{H},\left(\mathrm{C}_{6} \mathrm{H}_{3} \mathrm{~N}-\right.\right.$ $\left.\left.\mathrm{H}+\mathrm{CH}=\mathrm{NN}+3-\mathrm{FC}_{6} \mathrm{H}_{4}\right)\right], 7.21\left[\mathrm{~s}, 2 \mathrm{H},\left(\mathrm{CH}_{3} \mathrm{O}\right)_{3} \mathrm{C}_{6} \mathrm{H}_{2}\right]$, $3.72\left[\mathrm{~s}, 6 \mathrm{H},\left(\mathrm{OCH}_{3}\right)_{2}\right], 3.68\left(\mathrm{~s}, 3 \mathrm{H}, \mathrm{OCH}_{3}\right) ;{ }^{13} \mathrm{C} \mathrm{NMR}$ $\left(\mathrm{DMSO}-d_{6}, 125 \mathrm{MHz}\right) \delta: 170.3,163.8,161.8,154.2,153,6$, $152.5,149.8,148.9,148.8,140.5,139.8,135.3,133.9$, $132.3,131.3,128.6,127.9,126.3,121.4,121.2,115.5$, 106.2, 60.5, 56.6; IR (KBr) v: 3033, 2933, 2842, 1767, 1566, 1556, 1454, 1444, 1423, 1420, 1236, $750 \mathrm{~cm}^{-1}$. Anal. calcd for $\mathrm{C}_{27} \mathrm{H}_{20} \mathrm{Cl}_{2} \mathrm{FN}_{7} \mathrm{O}_{4} \mathrm{~S}$ : C 51.60, H 3.21, N 15.60; found $\mathrm{C} 51.76, \mathrm{H} 3.43, \mathrm{~N} 15.93$.

(E)-4-氯-2-(3-氯吡啶-2-基)-5-((4-((2,5-甲氧基苯基 次甲基)氨基)-5-(3,4,5-三甲氧基苯基)-4H-1,2,4-三唑-3基)硫基)-3(2H)-哒嗪酮(5h)：淡黄色粉末，产率 $38.3 \%$. 
m.p. $185 \sim 187{ }^{\circ} \mathrm{C} ;{ }^{1} \mathrm{H}$ NMR (DMSO- $\left.d_{6}, 500 \mathrm{MHz}\right) \delta: 9.02$ (s, $1 \mathrm{H}, \mathrm{CH}=\mathrm{N}), 8.56\left(\mathrm{~d}, J=4.00 \mathrm{~Hz}, 1 \mathrm{H}, \mathrm{C}_{6} \mathrm{H}_{3} \mathrm{NH}\right), 8.23$ $\left(\mathrm{d}, J=8.00 \mathrm{~Hz}, 1 \mathrm{H}, \mathrm{C}_{6} \mathrm{H}_{3} \mathrm{NH}\right), 7.77(\mathrm{~s}, 1 \mathrm{H}, \mathrm{CH}=\mathrm{NN})$, $7.65\left(\mathrm{t}, J=8.60 \mathrm{~Hz}, 1 \mathrm{H}, \mathrm{C}_{6} \mathrm{H}_{3} \mathrm{NH}\right), 7.51[\mathrm{~d}, J=8.00 \mathrm{~Hz}$, $\left.1 \mathrm{H},\left(\mathrm{CH}_{3} \mathrm{O}\right)_{2} \mathrm{C}_{6} \mathrm{H}_{3}\right], 7.31\left[\mathrm{~d}, J=8.05 \mathrm{~Hz}, 1 \mathrm{H},\left(\mathrm{CH}_{3} \mathrm{O}\right)_{2}-\right.$ $\mathrm{C}_{6} \mathrm{H}_{3}$ ], 7.20 [s, $\left.3 \mathrm{H},\left(\mathrm{CH}_{3} \mathrm{O}\right)_{2} \mathrm{C}_{6} \mathrm{H}_{3}+\left(\mathrm{CH}_{3} \mathrm{O}\right)_{3} \mathrm{C}_{6} \mathrm{H}_{2}\right], 3.82(\mathrm{~s}$, $\left.3 \mathrm{H}, \mathrm{OCH}_{3}\right), 3.72\left[\mathrm{~s}, 6 \mathrm{H},\left(\mathrm{OCH}_{3}\right)_{2}\right], 3.68\left(\mathrm{~s}, 3 \mathrm{H}, \mathrm{OCH}_{3}\right)$, $3.64\left(\mathrm{~s}, 3 \mathrm{H}, \mathrm{OCH}_{3}\right) ;{ }^{13} \mathrm{C}$ NMR (DMSO- $\left.d_{6}, 125 \mathrm{MHz}\right) \delta$ : $165.1,154.5,154.2,153.6,153.3,152.8,149.4,148.7$, $140.8,139.0,139.1,135.0,132.1,130.1,129.0,128.1$, $127.4,123.0,121.3,114.5,109.6,106.2,65.5,57.2,56.3$, 56.2; IR (KBr) v: 3050, 2960, 2837, 1730, 1668, 1591, 1573, 1494, 1477, 1427, 1238, $744 \mathrm{~cm}^{-1}$. Anal. calcd for $\mathrm{C}_{29} \mathrm{H}_{25} \mathrm{Cl}_{2} \mathrm{~N}_{7} \mathrm{O}_{6} \mathrm{~S}$ : C 51.95, H 3.76, N 14.62; found C 60.21, $\mathrm{H} 3.96, \mathrm{~N} 14.85$.

(E)-4-氯-2-(3-氯吡定-2-基)-5-((4- $((E)-((E)-3$-苯乙烯 基次甲基)氨基)-5-(3,4,5-三甲氧基苯基)- $4 H-1,2,4$-三唑3-基)硫基)-3(2H)-哒嗪酮(5i)：白色粉末，产率 39.8\%. m.p. $226 \sim 228{ }^{\circ} \mathrm{C} ;{ }^{1} \mathrm{H}$ NMR (DMSO- $d_{6}, 500 \mathrm{MHz}$ ) $\delta$ : $8.73(\mathrm{~d}, J=9.15 \mathrm{~Hz}, 1 \mathrm{H}, \mathrm{CH}=\mathrm{N}), 8.60(\mathrm{~d}, J=4.60 \mathrm{~Hz}$, $\left.1 \mathrm{H}, \mathrm{C}_{6} \mathrm{H}_{3} \mathrm{NH}\right), 8.27$ (d, J=8.00 Hz, $\left.1 \mathrm{H}, \mathrm{C}_{6} \mathrm{H}_{3} \mathrm{NH}\right), 7.76 \sim$ $7.78(\mathrm{~m}, 2 \mathrm{H}, \mathrm{CH}=\mathrm{CH}), 7.74(\mathrm{~s}, 1 \mathrm{H}, \mathrm{CH}=\mathrm{NN}), 7.69(\mathrm{t}$, $\left.J=8.05 \mathrm{~Hz}, 1 \mathrm{H}, \mathrm{C}_{6} \mathrm{H}_{3} \mathrm{NH}\right), 7.36 \sim 7.51\left(\mathrm{~m}, 5 \mathrm{H}, \mathrm{C}_{6} \mathrm{H}_{5} \mathrm{H}\right)$, $7.21\left[\mathrm{~s}, 2 \mathrm{H},\left(\mathrm{CH}_{3} \mathrm{O}\right)_{3} \mathrm{C}_{6} \mathrm{H}_{2}\right], 3.81\left[\mathrm{~s}, 6 \mathrm{H},\left(\mathrm{OCH}_{3}\right)_{2}\right], 3.74(\mathrm{~s}$, $3 \mathrm{H}, \mathrm{OCH}_{3}$ ); ${ }^{13} \mathrm{C}$ NMR (DMSO- $\left.d_{6}, 125 \mathrm{MHz}\right) \delta: 173.7$, $154.3,153.3,152.3,150.2,149.5,148.7,140.4,140.2$, $139.7,135.0,131.4,130.8,129.6,129.0,128.5,127.8$, 122.9, 121.9, 121.6, 106.3, 105.7, 60.7, 56.5; IR (KBr) v: 3019, 2922, 1770, 1625, 1577, 1556, 1519, 1479, 1423, 1296, 1240, $723 \mathrm{~cm}^{-1}$. Anal. calcd for $\mathrm{C}_{29} \mathrm{H}_{23} \mathrm{Cl}_{2} \mathrm{~N}_{7} \mathrm{O}_{4} \mathrm{~S}: \mathrm{C}$ 54.72, H 3.64, N 15.40; found C 54.92, H 3.87, N 15.71 .

(E)-4-氯-2-(3-氯吡啶-2-基)-5-((4-((2-氯苯基次甲基) 氨基)-5-(3,4,5-三甲氧基苯基)-4H-1,2,4-三唑-3-基)硫 基)-3(2H)-挞嗪酮 $(\mathbf{5 j})$ : 淡黄色粉末, 产率 35.8\%. m.p. 231 233 ${ }^{\circ} \mathrm{C} ;{ }^{1} \mathrm{H}$ NMR (DMSO- $\left.d_{6}, 500 \mathrm{MHz}\right) \delta: 9.14$ (s, $1 \mathrm{H}, \mathrm{CH}=\mathrm{N}), 8.56\left(\mathrm{~d}, J=4.55 \mathrm{~Hz}, 1 \mathrm{H}, \mathrm{C}_{6} \mathrm{H}_{3} \mathrm{NH}\right), 8.23(\mathrm{~d}$, $\left.J=6.90 \mathrm{~Hz}, 1 \mathrm{H}, \mathrm{C}_{6} \mathrm{H}_{3} \mathrm{NH}\right), 8.08(\mathrm{~d}, J=7.45 \mathrm{~Hz}, 1 \mathrm{H}$, $\left.2-\mathrm{ClC}_{6} \mathrm{H}_{4}\right), 7.78(\mathrm{~s}, 1 \mathrm{H}, \mathrm{CH}=\mathrm{NN}), 7.61 \sim 7.67(\mathrm{~m}, 3 \mathrm{H}$, 2- $\left.\mathrm{ClC}_{6} \mathrm{H}_{4}+\mathrm{C}_{6} \mathrm{H}_{3} \mathrm{NH}\right), 7.51\left(\mathrm{t}, J=7.45 \mathrm{~Hz}, 1 \mathrm{H}, 2-\mathrm{ClC}_{6} \mathrm{H}_{4}\right)$, 7.19 [s, 2H, $\left(\mathrm{CH}_{3} \mathrm{O}\right)_{3} \mathrm{C}_{6} \mathrm{H}_{2}$ ], 3.73 [s, 6H, $\left.\left(\mathrm{OCH}_{3}\right)_{2}\right], 3.69$ (s, $3 \mathrm{H}, \mathrm{OCH}_{3}$ ); ${ }^{13} \mathrm{C}$ NMR (DMSO- $\left.d_{6}, 125 \mathrm{MHz}\right) \delta: 166.0$, $154.2,153.5,152.8,149.6,148.7,140.4,140.2,139.9$, $139.3,136.0,135.7,134.9,131.1,130.7,129.1,128.8$, 128.6, 128.5, 127.9, 121.6, 106.7, 60.8, 56.6; IR (KBr) v: 3061, 2922, 1739, 1676, 1589, 1558, 1479, 1456, 1423,
1236, $1132,771 \mathrm{~cm}^{-1}$. Anal. calcd for $\mathrm{C}_{27} \mathrm{H}_{20} \mathrm{Cl}_{3} \mathrm{~N}_{7} \mathrm{O}_{4} \mathrm{~S}$ : C 54.72, H 3.64, N 15.40; found C 54.92, H 3.87, N 15.71 .

\section{2 结果与讨论}

\section{1 合成及机理}

\subsection{1 合成}

中间体 2-肼基-3-氯吡啶(3)的合成, 采用 $80 \%$ 水合 肼溶液既作溶剂又作反应物，在短时间下得到理想的产 率. 在中间体 2-(3-氯吡啶-3-基)-4,5-二氯-3(2H)-哒嗪酮 (4)的合成上, 发现加入催化量 $5 \%$ 稀盐酸溶液作催化剂 能在一定程度上缩短反应时间(加入催化量 $5 \%$ 稀盐酸溶 液作催化剂, 回流 $3 \mathrm{~h}$, 反应完全, 不加入盐酸反应亦能 进行, 但反应完全则需要 $6 \mathrm{~h}$ ).

目标化合物 5 的合成, 以目标化合物 $\mathbf{5 e}$ 的合成为 例, 采用了乙腈/碳酸钾体系, 常温下搅拌约 $6 \mathrm{~h}$, 反应 完全, TLC 跟踪发现有两种产物生成, 柱层析分离得目 标化合物 5e 和双取代的副产物 6, 之后在同样的反应条 件下, 将温度升至 $45{ }^{\circ} \mathrm{C}$, 搅拌约 $2 \mathrm{~h}$, 反应完全, 缩短 了反应时间, 但副产物 6 的量没有太多的变化, 据此确 定了合成方法，该方法反应条件温和、后处理简单. 副 产物 6 的形成过程如 Eq. 1, 数据表征如下: 黄色粉末, 产率 30.9\%. m.p. $168 \sim 170{ }^{\circ} \mathrm{C} ;{ }^{1} \mathrm{H}$ NMR (DMSO- $d_{6}, 500$ MHz) $\delta: 8.99(\mathrm{~s}, 1 \mathrm{H}, \mathrm{CH}=\mathrm{N}), 8.96(\mathrm{~s}, 1 \mathrm{H}, \mathrm{CH}=\mathrm{N}), 8.51$ $\left(\mathrm{d}, J=4.60 \mathrm{~Hz}, 1 \mathrm{H}, \mathrm{C}_{6} \mathrm{H}_{3} \mathrm{NH}\right), 8.16(\mathrm{~d}, J=6.85 \mathrm{~Hz}, 1 \mathrm{H}$, $\left.\mathrm{C}_{6} \mathrm{H}_{3} \mathrm{NH}\right), 7.85(\mathrm{~s}, 1 \mathrm{H}, \mathrm{CH}=\mathrm{NN}), 7.58 \sim 7.61(\mathrm{~m}, 1 \mathrm{H}$, $\left.\mathrm{C}_{6} \mathrm{H}_{3} \mathrm{NH}\right), 7.35 \sim 7.36\left[\mathrm{~m}, 2 \mathrm{H},\left(\mathrm{CH}_{3} \mathrm{O}\right)_{2} \mathrm{C}_{6} \mathrm{H}_{3}\right], 7.18[\mathrm{~d}, J=$ $\left.2.85 \mathrm{~Hz}, 1 \mathrm{H},\left(\mathrm{CH}_{3} \mathrm{O}\right)_{2} \mathrm{C}_{6} \mathrm{H}_{3}\right], 7.16\left[\mathrm{~s}, 2 \mathrm{H},\left(\mathrm{CH}_{3} \mathrm{O}\right)_{3} \mathrm{C}_{6} \mathrm{H}_{2}\right]$, $7.15\left[\mathrm{~d}, J=3.45 \mathrm{~Hz}, 1 \mathrm{H},\left(\mathrm{CH}_{3} \mathrm{O}\right)_{2} \mathrm{C}_{6} \mathrm{H}_{3}\right], 7.09[\mathrm{~s}, 2 \mathrm{H}$, $\left.\left(\mathrm{CH}_{3} \mathrm{O}\right)_{3} \mathrm{C}_{6} \mathrm{H}_{2}\right], 7.07$ [d, $\left.J=9.20 \mathrm{~Hz}, 1 \mathrm{H},\left(\mathrm{CH}_{3} \mathrm{O}\right)_{2} \mathrm{C}_{6} \mathrm{H}_{3}\right]$, $7.04\left[\mathrm{~d}, J=9.75 \mathrm{~Hz}, 1 \mathrm{H},\left(\mathrm{CH}_{3} \mathrm{O}\right)_{2} \mathrm{C}_{6} \mathrm{H}_{3}\right], 3.71[\mathrm{~s}, 6 \mathrm{H}$, $\left.\left(\mathrm{OCH}_{3}\right)_{2}\right], 3.69$ [s, 6H, $\left.\left(\mathrm{OCH}_{3}\right)_{2}\right], 3.68\left[\mathrm{~s}, 6 \mathrm{H},\left(\mathrm{OCH}_{3}\right)_{2}\right]$, $3.67\left(\mathrm{~s}, 3 \mathrm{H}, \mathrm{OCH}_{3}\right), 3.66\left[\mathrm{~s}, 6 \mathrm{H},\left(\mathrm{OCH}_{3}\right)_{2}\right], 3.65(\mathrm{~s}, 3 \mathrm{H}$, $\mathrm{OCH}_{3}$ ); ${ }^{13} \mathrm{C}$ NMR (DMSO- $\left.d_{6}, 125 \mathrm{MHz}\right) \delta: 165.1,164.2$, $155.2,154.9,153.7,153.5,152.3,150.9,149.7,148.6$, $142.4,142.0,140.5,140.3,139.6,139.5,135.9,131.6$, $128.6,127.7,123.0,122.9,121.8,121.7,120.0,119.9$, 114.8, 114.7, 109.9, 109.7, 106.4, 105.8, 60.7, 56.9, 56.4, 56.4, 56.1; IR (KBr) v: 3013, 2913, 1714, 1660, 1587, 1496, 1458, 1427, 1350, 1224, 1126, $713 \mathrm{~cm}^{-1}$. Anal. calcd for $\mathrm{C}_{49} \mathrm{H}_{46} \mathrm{ClN}_{11} \mathrm{O}_{11} \mathrm{~S}_{2}$ : C 55.28, H 4.36, N 14.47; found C 54.89, H 4.69, N 14.29.

2.1.2 机理

合成中间体 4 可能的反应机理见 Scheme 2. 首先化 合物 2-肼基-3-氯吡啶(3)中与吡啶环相连的 $\beta$-氮原子进 
<smiles></smiles><smiles>COc1cccc(/C=N/n2c(S)nnc2-c2cc(OC)c(OC)c(OC)c2)c1OC</smiles><smiles>COc1cccc(/C=N/n2c(Sc3c(Sc4nnc(-c5cc(OC)c(OC)c(OC)c5)n4/N=C/c4cccc(OC)c4OC)cnn(-c4ncccc4Cl)c3=O)nnc2-c2cc(OC)c(OC)c(OC)c2)c1</smiles>

6 By-product

攻糠氯酸中的醛羰基发生亲核加成反应得到化合物 8 (此过程经历过渡态 7), 然后化合物 8 脱水形成具有腙 结构的化合物 9, 最后化合物 9 在酸的催化作用下脱水 成环得到 4.<smiles>[Z7]C(NNc1ncccc1Cl)C([O-])[C@@H](Cl)C(Cl)=C(Cl)C(=O)O</smiles>

3<smiles>COC(=O)C(Cl)=C(Cl)C(O)NNc1ncccc1Cl</smiles>

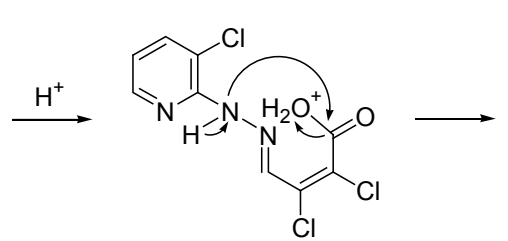

10<smiles>O=C(O)/C(Cl)=C(/O)C(O)=NNc1ncccc1Cl</smiles><smiles>O=c1c(Cl)c(Cl)cnn1-c1ncccc1Cl</smiles>

Scheme 2<smiles>[R]C=Nn1c(S)nnc1-c1cc(OC)c(OC)c(OC)c1</smiles>

2
合成目标化合物 $\mathbf{5}$ 的可能反应机理见 Scheme 3: 首 先化合物 2 与碳酸钾反应生成钾盐 11 , 然后化合物 11 与 4 发生亲核取代反应得到目标化合物 $\mathbf{5}$.

\section{2 红外光谱和核磁共振氢谱分析}

IR 中, 化合物 1 与醛缩合生成化合物 $\mathbf{2}$, 出现亚胺 $\mathrm{C}=\mathrm{N}\left(1620 \mathrm{~cm}^{-1}\right)$ 吸收. 化合物 $\mathbf{2}$ 与 $\mathbf{4}$ 反应生成 $\mathbf{5}$ 之后, 在 750,1730 及 $1660 \mathrm{~cm}^{-1}$ 附近出现 $\mathrm{C}-\mathrm{S}-\mathrm{C}, \mathrm{C}=\mathrm{O}$ 和 $\mathrm{C}=\mathrm{N}$ 的吸收峰.

${ }^{1} \mathrm{H}$ NMR 中，目标化合物 5 有共同的亚胺结构 $(\mathrm{CH}=\mathrm{N})($ 氢在 $\delta 9$ 左右)、哒嗪酮环上唯一的氢(氢以单 峰的形式出现在 $\delta 7.7$ 左右)及与哒嗪酮环相连的吡啶环 上三个氢(三个氢分别以双峰、双峰及三重峰的形式出 现在 $\delta 7 \sim 8$ 之间), 且无化合物 2(以硫酮形式存在)三唑 环上质子 $(\mathrm{NH})$ (化学位移在 $\delta 14$ 左右), 说明化合物 2 与 4 之间发生了反应.

\section{3 生物活性测试}

\subsection{1 实验方法}

采用生长速率法, 以小麦赤霉菌 $(G . z e a e)$ 、苹果腐 烂病菌 $(C$. mandshurica $)$ 和辣椒枯萎病菌 $(F$. oxysporum $)$ 为供试对象, 对供试化合物的抑菌活性进行初步篎选. 主要采用马铃薯葡萄糖琼脂(PDA)培养基, 分别量取 90 $\mathrm{mL}$ 培养基分装在 $200 \mathrm{~mL}$ 三角瓶中灭菌备用. 含药培养 基的制备均在无菌条件下进行，每种药剂浓度设为 50 $\mathrm{mg} / \mathrm{L}$. 分别称取各种药剂于 $10 \mathrm{~mL}$ 容量瓶中, 加入含 $0.5 \%$ Tween 20 的灭菌水配制成一定浓度的药剂, 加入 $90 \mathrm{~mL}$ PDA 培养基(40 50 $\left.{ }^{\circ} \mathrm{C}\right)$ 中, 充分摇匀, 将其倒入 直径 $9 \mathrm{~cm}$ 的灭菌后的培养血中, 设三次重复, 以加入等 量的溶剂为空白对照. 初笁时, 以小麦赤霉病菌、黄瓜 灰霉病菌、油菜菌核病菌等为笁选对象, 以打孔器(内径 $4 \mathrm{~mm}$ ) 将生长正常的菌落打孔制成若干菌饼备用，用接 种针将菌饼移接到平板中央, 每皿接一个菌饼, 置于 27 ${ }^{\circ} \mathrm{C}$ 饱和湿度恒温培养箱中培养, 待对照长满时, 测量 菌落直径. 每个菌落按十字交叉法测量 2 次, 以其平均 数代表菌落的大小, 抑菌率的计算公式如下:

Scheme 3 
抑制率 $=\frac{\text { 对照菌落直径一处理菌落直径 }}{\text { 对照菌落直径 }-0.4} \times 100 \%$

\subsection{2 实验结果}

生物活性测试结果见表 1 .

表 1 目标化合物 5 的抑菌活性(抑制率/\%)

Table 1 Antifungal activities of target compound $\mathbf{5}$ (percent inhibition/\%)

\begin{tabular}{cccc}
\hline Compound & G. zeae & C. mandshurica & F. oxysporum \\
\hline $\mathbf{5 a}$ & $41.5 \pm 1.8$ & $9.32 \pm 1.8$ & $17.55 \pm 1.1$ \\
$\mathbf{5 b}$ & $46.4 \pm 3.0$ & $5.02 \pm 1.7$ & $19.54 \pm 0.8$ \\
$\mathbf{5 c}$ & $5.64 \pm 0.82$ & $8.63 \pm 1.3$ & $5.94 \pm 0.81$ \\
$\mathbf{5 d}$ & $29.97 \pm 1.0$ & $9.32 \pm 1.7$ & $21.85 \pm 1.2$ \\
$\mathbf{5 e}$ & $14.24 \pm 1.01$ & $12.95 \pm 1.1$ & $10.94 \pm 1.00$ \\
$\mathbf{5 f}$ & $16.91 \pm 1.25$ & $9.71 \pm 1.3$ & $17.81 \pm 1.32$ \\
$\mathbf{5 g}$ & $16.62 \pm 0.79$ & $13.30 \pm 1.3$ & $18.44 \pm 1.05$ \\
$\mathbf{5 h}$ & $6.53 \pm 1.09$ & $4.68 \pm 1.2$ & $3.13 \pm 0.83$ \\
$\mathbf{5 i}$ & $19.31 \pm 1.9$ & $5.02 \pm 1.7$ & $15.23 \pm 0.9$ \\
$\mathbf{5 j}$ & $1.19 \pm 0.77$ & $6.11 \pm 1.3$ & $1.56 \pm 0.71$ \\
Carbendazim & $100 \pm 5.4$ & $100 \pm 6.0$ & $100 \pm 5.9$ \\
\hline
\end{tabular}

由表 1 可见, 在 $50 \mathrm{mg} / \mathrm{L}$ 的浓度下, 化合物 $\mathbf{5}$ 对小 麦赤霉病菌、苹果腐烂病菌和辣椒枯萎病菌有一定的抑 制作用, 其中 5a 与 5b 对小麦赤霉病菌的抑制率分别可 达到 41.5\%, 46.4\%, 且对小麦赤霉病菌的抑制作用总体 上来说明显要好于对苹果腐烂病菌与辣椒枯萎病菌的.

\section{References}

[1] Cao, L.- H.; Wang, C.- F.; Tao, J. Chin. J. Org. Chem. 2006, 26, 1686 (in Chinese)

(曹玲华, 王彩芳, 陶晶, 有机化学, 2006, 26, 1686.)
[2] Bai, K.; Yao, C.- P.; Pei, W. Chin. J. Org. Chem. 2010, 30, 1255 (in Chinese). (拜斿, 姚彩萍, 裴文, 有机化学, 2010, 30, 1255.)

[3] Wu, J.; Song, B.-A.; Chen, H.-J. Molecules 2009, 14, 3676.

[4] Sun, X.-H.; Tao, Y.; Liu, Y.-F. Acta Chim. Sinica 2008, 66, 234 (in Chinese).

(孙晓红, 陶燕, 刘源发, 化学学报, 2008, 66, 234.)

[5] Li, Y.-J.; Liu, L.-J.; Jin, K. Acta Chim. Sinica 2010, 68, 1577 (in Chinese).

(李英俊, 刘丽军, 靳焜, 化学学报, 2010, 68, 1577.)

[6] Suresh Kumar, G. V.; Rajendraprasad, Y.; Mallikarjuna, B. P. Eur. J. Med. Chem. 2010, 45, 2063.

[7] Bagrak, H.; Demirbas, A.; Demirbas, N. Eur. J. Med. Chem. 2009 , 44, 1057.

[8] Xu, S.-Z.; Ming, Z.-H.; Yang, J.-Y. Chin. J. Org. Chem. 2010, 30, 285 (in Chinese). (徐胜臻, 明志会, 杨娇绝, 有机化学, 2010, 30, 285.)

[9] Yang, Z.-K.; Zhang, H.-K.; Fan, Z.-K. Chin. J. Pestic. Sci. 2009, 11, 19 (in Chinese). (杨知昆, 张海科, 范志全, 农药学学报, 2009, 11, 19.)

[10] Sun, X.-H.; Bai, Y.; Liu, Y.-F. Chem. J. Chin. Univ. 2011, 32, 1312 (in Chinese). (孙晓红, 白燕, 刘源发, 高等学校化学学报, 2011, 32, 1312.)

[11] Tian, Z.-Z.; Jiang Z.-X.; Li, Z.; Song, G. H. J. Agric. Food Chem. 2007, 55, 143.

[12] MKarthikeyan, M. S.; Prasad, D. J.; Poojary, B. Bioorg. Med. Chem. 2006, 14, 7482 .

[13] Li, Q.-Z.; Song, B.-A.; Cai, X.-J. Chin. J. Org. Chem. 2010, 30, 569 (in Chinese). (李黔柱, 宋宝安, 蔡学健, 有机化学, 2010, 30, 569.)

[14] Zheng, Y.-G.; Wei, X.; Xue, W. Chem. Reag. 2011, 33, 209 (in Chinese). (郑玉国, 魏学, 薛伟, 化学试剂, 2011, 33, 209.)

[15] Chai, B.-S.; Peng, Y.-W.; Liu, C.-L. Agrochemicals 2009, 48, 13 (in Chinese). (柴宝山, 彭永武, 刘长令, 农药, 2009, 48, 13.) 